\title{
Cäsar Adolf Bloesch - ein Geburtshelfer? Einblicke in seine ärztliche Praxis zwischen 1832 und 1850
}

Philipp Klaas

\section{Summary}

This article contributes to the history of obstetrics as it was performed in the context of general practice during the 19th century. It focuses on the Swiss physician Cäsar Adolf Bloesch (1804-1863) from the town of Biel, Canton Bern. Drawing upon Bloesch's extensive practice records, it raises the question whether this physician participated in the local market for obstetrical activities. Furthermore, the paper tries to make an estimate about the importance this field of action took for Bloesch's practice. To achieve this, results of a smaller study about obstetrical cases within the practice records during the years 1832 to 1850 are being presented. I will argue that physicians like Bloesch were aware of the problems obstetrics brought with it, and as a consequence, he tried to limit it in favor of his general practice.

Keywords: Cäsar Adolf Bloesch (1804-1863), medical diary, midwifery, obstetrics, 19th century

\section{Zusammenfassung}

Der folgende Artikel versteht sich als Beitrag zur Geschichte der privatärztlichen Geburtshilfe im 19. Jahrhundert. Im Mittelpunkt steht die Praxis des schweizerischen Arztes Cäsar Adolf Bloesch (1804-1863), der von 1827 bis 1863 in seiner Heimatstadt Biel im Kanton Bern aktiv war. Er geht der Frage nach, ob und in welcher Form sich der Arzt Bloesch als aktiver Geburtshelfer betätigte und welche Bedeutung dieser Tätigkeitsbereich

Philipp Klaas, M.A., Institut für Medizingeschichte, Bühlstraße 26, CH-3012 Bern (philipp.klaas @img.unibe.ch). 
schlussendlich für sein Wirken als Arzt am lokalen medizinischen Markt hatte. Hierfür sollen Ergebnisse bisheriger Recherchen präsentiert werden, die sich auf Bloeschs umfangreiches Praxisjournal stützen und den Zeitraum zwischen 1832 und 1850 abdecken. Es zeigt sich, dass die Geburtshilfe Ärzte wie Bloesch vor ein grundlegendes Dilemma stellte: Zwar herrschte im städtischen Umfeld eine grundsätzliche Nachfrage, doch fürchteten sie zugleich die negativen Folgen, die ihr Engagement für die Allgemeinpraxis haben konnte.

Schlagworte: Cäsar Adolf Bloesch (1804-1863), Praxisjournal, Geburtshilfe, 19. Jahrhundert

Die (medizin-)historische Forschung ist heute mehr und mehr darauf bedacht, die «Medikalisierung der Geburt» differenzierter zu betrachten. ${ }^{1}$ Ein Grund dafür ist der zunehmende Attraktivitätsverlust, den das lange weitverbreitete Narrativ einer auf breiter Front betriebenen «Entmündigung» und «Disziplinierung» von werdenden Müttern und Hebammen durch die männlichakademische Medizin mittlerweile erfahren hat. ${ }^{2}$ Noch aber gibt es viele Forschungsdesiderate in diesem Bereich. ${ }^{3}$ So fehlt es beispielsweise für das 19. Jahrhundert noch immer weitgehend an Untersuchungen über den Arbeitsalltag von Hebammen und akademischen Ärzten, den beiden wichtigsten Anbietern von professioneller Geburtshilfe. ${ }^{4}$ Gerade mit ihrer Hilfe ließen sich aber viele weitere Aufschlüsse darüber gewinnen, in welchem Umfang Ärzte und Hebammen Geburtsmedizin betrieben, welche sozialen Faktoren ihr Tun beeinflussten und wie sie mit den Patientinnen interagierten.

Im folgenden Beitrag möchte ich einige Ergebnisse meiner laufenden Untersuchungen zur Geburtshilfe im Rahmen der Praxis von Cäsar Adolf Bloesch vorstellen, der von 1827 bis zu seinem Tod im Jahr 1863 als Arzt in der Stadt Biel im Kanton Bern wirkte. Hauptquelle der Untersuchung ist das von Bloesch angefertigte Praxisjournal, dessen erhaltene Bände die zwischen 1832 und 1863 geleistete Arbeit erfassen. ${ }^{5}$ Meine Recherchen

1 Dieser Beitrag entstand im Rahmen des vom SNF geförderten Forschungsprojektes «Die Praxis des Bieler Arztes Cäsar Adolf Bloesch (1804-1863)» am Institut für Medizingeschichte der Universität Bern. Für Kritik und Anregungen danke ich Hubert Steinke und Markus Binder.

2 Vgl. hierzu Gross 1998 und Green 2008.

3 Vgl. zusammenfassend Ellerbrock 2001, 124.

4 Vgl. Seidel 1998, 25, 28-30, und den Beitrag von Marina Hilber in diesem Band.

5 Die ersten beiden Journalbände sind verloren. Die medizinischen Aufzeichnungen beginnen im dritten Band im Juni 1832, wodurch eine Überlieferungslücke von fast fünf Jahren entsteht. Vor den Praxisaufzeichnungen finden sich zudem buchhalterische Einträge, die bis 1828 zurückreichen. Vgl. Boucherin 2008, 148. 
erstreckten sich über den Zeitraum von 1832 bis 1850 und deckten damit sowohl die Frühphase der Praxis als auch die Zeit ab, in der Bloesch seine Position am medizinischen Markt seiner Heimatstadt weitgehend gefestigt hatte. Welche Rolle hierbei die Geburtshilfe spielte, soll im Folgenden erörtert werden. Denn obgleich die ärztliche Geburtshilfe seit ihrem Aufstieg im 18. Jahrhundert inner- wie außerhalb der Medizin stets kontrovers diskutiert wurde, war sie gerade für junge Mediziner ab 1800 oft eine Möglichkeit, eine eigene Praxis aufzubauen. ${ }^{6}$ Durch erfolgreiche Hilfe am Wochenbett ließ sich vor allem das Zutrauen der weiblichen Klientel gewinnen, was besonders wichtig werden konnte, wenn es sich dabei um Hausfrauen handelte. Waren sie als «Gesundheitshüterinnen der Familie» mit den Leistungen des Arztes zufrieden, erhöhte dies günstigenfalls die Chancen, in Zukunft sukzessive auch bei Gesundheitsproblemen der anderen Familienmitglieder herangezogen zu werden. ${ }^{7}$ Darüber hinaus wurde die Geburtshilfe generell im Lauf des 19. Jahrhunderts von Patientenseite immer häufiger und selbstverständlicher in Anspruch genommen, vor allem von wohlhabenden Klienten wie dem gehobenen städtischen Bürgertum. ${ }^{8}$

Im Folgenden möchte ich mich den beiden Fragen widmen, ob und in welcher Form sich der Arzt Cäsar Adolf Bloesch in der Geburtshilfe engagierte und welche Bedeutung dieses Tätigkeitsfeld für seine Praxis gehabt hat. In einem ersten Schritt wird es darum gehen, den geburtshilflichen Markt der Stadt Biel darzustellen und den Arzt Bloesch im Feld der approbierten Anbieter zu verorten. Danach werde ich anhand ausgewählter Beispiele darstellen, wie der Arzt Bloesch sich der Geburtshilfe widmete. Zum Schluss werden die Befunde zusammengefasst und diskutiert.

\section{Biels Geburtsmarkt um 1840}

Noch bevor sich Cäsar Adolf Bloesch 1827 nach Studienaufenthalten in Göttingen, Berlin und Paris als Arzt in seiner Heimatstadt niederlie $\beta,{ }^{9}$ dürfte ihm klar gewesen sein, dass der Berufseinstieg an diesem Ort eine große Herausforderung sein würde. Allem Anschein nach war Biel in jenen Tagen

6 Vgl. Digby 1994,254. Den Unterschied zum 18. Jahrhundert stellen heraus Rieder/Louis-Courvoisier 2010, 587.

7 Vgl. Porter/Porter 1989, S. 183; Seidel 1998, S. 334f.

8 Vgl. Seidel 1998,381f., 400. Digby schätzt, dass englische Ärzte im 19. Jahrhundert bis zu einem Drittel ihres Einkommens aus der Geburtshilfe erwirtschafteten, wobei diejenigen mit einer exklusiveren Patientenschaft weniger auf diesen Erwerbszweig angewiesen waren als andere Kollegen. Vgl. Digby 1994, 257.

9 Als biographischen Überblick vgl. Bourquin/Bourquin 2008, Artikel «Bloesch», 77f. 
durchaus attraktiv für Heilkundige, was nicht zuletzt auch auf die dynamische Stadtentwicklung zurückgeführt werden kann. Als Sitz der Verwaltung des gleichnamigen Amtsbezirks, als Marktort und gewerblich-industrielles Zentrum der Region mit einer großen Vielfalt eingesessener Handwerksund Manufakturbetriebe vereinigte Biel bei aller Überschaubarkeit der Verhältnisse wichtige Funktionen auf sich. Bis 1837 stieg der Ort mit 2796 Einwohnern immerhin zur drittgrößten Stadt ${ }^{10}$ im Kanton auf, wenngleich der Schritt zu Urbanisierung und gesteigerter Expansion erst nach 1850 erfolgte. ${ }^{11}$ Gerade Ärzte scheinen die potentielle Nachfrage nach medizinischen Leistungen aus Biel und dem relativ dicht bewohnten Umland ${ }^{12}$ sowie ihre Verdienstmöglichkeiten als vielversprechend eingeschätzt zu haben: Seit Bloeschs Niederlassung verdoppelte sich ihre Zahl innerhalb von 15 Jahren von vier auf acht. ${ }^{13}$ Neben der steigenden Konkurrenz kam erschwerend hinzu, dass er selbst - anders als manch andere junge Mediziner - zu Beginn seiner Laufbahn nicht in der glücklichen Position war, eine bestehende Praxis übernehmen zu können. Zwar hatte bereits sein Vater Johann Alexander Bloesch in der Stadt als Arzt gewirkt, durch dessen frühzeitigen Tod war der Sohn aber gezwungen, sich bald einen eigenen Kundenkreis aufzubauen. ${ }^{14}$ In ähnlichen Situationen entschieden sich junge Praktiker in der ersten Hälfte des 19. Jahrhunderts immer häufiger für die Geburtshilfe als Mittel, um neue Patientinnen sowie die Aufmerksamkeit und das Zutrauen des jeweiligen Familienumfeldes zu gewinnen. Die Chancen, sich mit diesem Angebot profilieren zu können, waren allgemein gut, da etablierte ältere Kollegen die Obstetrik oft noch gar nicht oder nur selten ausübten..$^{15}$ Wie das Beispiel Jakob Büchlers zeigt, konnte das Geburtsgeschäft auch für junge Ärzte im Kanton der Schlüssel zu einer ansehnlichen Tätigkeit sein: Nachdem er 1829 seine Arbeit im Juradorf Renan aufgenommen hatte, durfte er sich nach eigenen Angaben schon nach den ersten zwölf Monaten einer «beträchtlichen Praxis» erfreuen. Die Geburtshilfe habe darin «nicht den geringsten Theil» ausgemacht, so dass er innerhalb

10 Vgl. Hildebrand 1860, 61.

11 Vgl. von Niederhäusern 2011, 72.

12 Vgl. Pfister/Egli 1998, 46f.

13 Unklar ist aber, ob sie alle primär in Biel wirkten: Laut amtlichem Verzeichnis waren 1842 alle acht Ärzte des Amtsbezirks Biel in der Stadt Biel wohnhaft. Für sieben von ihnen ist es sehr wahrscheinlich, dass ihr Wohn- mit dem Arbeitsort zusammenfiel. Der aus Württemberg stammende Bernhard Carl Finkbeiner führte aber neben der Privatpraxis auch eine eigene Irrenanstalt im nahegelegenen Dorf Mett, das zum Amtsbezirk Nidau gehörte. Ob er in Biel ein Behandlungslokal besaß, ist unbekannt. Vgl. Schneider/Emmert 1842, 4, 6, und Mahlberg 2005, S. 51.

$14 \mathrm{Vgl}$. Bourquin/Bourquin 2008, Artikel «Bloesch», 77. Zu den Schwierigkeiten des ärztlichen Berufseinstiegs vgl. Huerkamp 1985, 122-125.

15 Vgl. Seidel 1998, 335. 
von zehn Jahren zu 379 Geburten gerufen worden sei. Wenngleich Büchler hierunter auch solche Fälle gezählt hatte, in denen er «nach vorläufigem Touchiren bloß Rath ertheilt» habe, führte er im Schnitt immerhin sechs künstliche Geburten pro Jahr durch, womit er als gutbeschäftigt gelten darf. ${ }^{16}$

Die Nachfrage nach professionell geschulter Hilfe am Wochenbett scheint in der Region also durchaus vorhanden gewesen zu sein, wenngleich Ärzte nicht die einzigen Anbieter auf diesem Gebiet waren. Nachdem der Kanton 1781 aus bevölkerungspolitischen Erwägungen heraus erstmals eine eigene Schule für die theoretische und praktische Ausbildung von Hebammen ins Leben gerufen hatte, absolvierten während des 19. Jahrhunderts immer mehr Bewerberinnen die in der Hauptstadt stattfindenden Kurse. ${ }^{17}$ Anfängliche Vorbehalte in der Bevölkerung, die viele der hier unterwiesenen Frauen bald wieder zur Berufsaufgabe zwangen, scheinen sich im Lauf der Zeit verloren zu haben. ${ }^{18}$ Neben den Absolventinnen der Hebammenschule und solchen, die nach der Lehrzeit bei einem Arzt die Prüfung vor dem Sanitätskollegium bestanden, ${ }^{19}$ wirkten jedoch weiterhin unpatentierte Geburtshelferinnen im Kanton. Über ihre Zahl ist aber nichts Genaues bekannt. ${ }^{20}$ Wie in vielen anderen Territorien des deutschsprachigen Raumes üblich, waren auch approbierte bernische Hebammen in erster Linie für die Betreuung «normaler», sprich komplikationslos verlaufender Geburten verantwortlich. Daneben oblag ihnen vor allem die Pflege von Schwangeren, Wöchnerinnen und Neugeborenen. ${ }^{21}$ Die Kompetenzen und Vorrechte der übrigen Heilergruppen setzten der Berufsausübung der Hebammen aber stets Grenzen, die es zu berücksichtigen galt. Bei schwierigen Entbindungen sollten sie unverzüglich einen «geschickte[n] Geburtshelfer» hinzuziehen und diesem nach seinem Eintreffen die weitere Behandlung abtreten. Auch bei inneren und äußeren Krankheiten von Mutter und Kind, die über gelinde

16 Hierzu zählen 36 Zangengeburten, 24 Kindswendungen und der dreimalige Einsatz des Geburtshakens. Dies entsprach jenen Zahlen, die auch württembergische Geburtshelfer im 19. Jahrhundert erreichten. Nach Seidels Einschätzung waren 10 bis 30 jährliche Entbindungen für einen Arzt im ländlich-kleinstädtischen Umfeld bereits viel. Siehe und vgl. Büchler 1842, 101-104, und Seidel 1998, 339.

17 Vgl. Jenzer 1966, 70, und Meyer 1985.

18 Vgl. Meyer 1985, 47, 57. Nach einem Verwaltungsbericht über die ersten sechs Jahre der Hebammenschule gab ein Drittel der 102 examinierten Hebammen ihren Beruf wieder auf, weil er ihnen «weder Brod noch Zuneigung verschaffete». Zitat nach ebd., 58. Zu den vielschichtigen Konflikten zwischen approbierten Hebammen und lokaler Bevölkerung seit dem 18. Jahrhundert vgl. Labouvie 1999, S. 218-234.

19 Vgl. Meyer 1985, S. 80.

20 Den Berichten Jakob Büchlers zufolge traf er bei seiner Arbeit ziemlich regelmäßig auf ungeprüfte Hebammen. Vgl. Flückiger/Marthaler 2003, 15.

21 Siehe und vgl. Schneider/Emmert 1842, 43; Schulz 2003, 20f. Zu den normativen Hierarchien zwischen beiden Berufsgruppen und deren Folgen für die soziale Praxis vgl. Seidel 1998, $150-155$. 
verlaufende Wochenbetterkrankungen hinausgingen, waren Hebammen gehalten, eigene Kurversuche zu unterlassen. Überhaupt galt, dass Ärzte ein grundsätzliches Entscheidungs- und Behandlungsmonopol genossen, sobald sie aus irgendeinem Anlass «zur Besorgung einer Schwangern, Gebärenden oder Wöchnerinn gerufen» wurden. Die weiblichen Geburtshelferinnen hatten in diesem Fall - selbst wenn man sich zuerst an sie gewandt hatte den ärztlichen Anweisungen zu folgen. Das Halten einer Hausapotheke und das Verordnen von Arzneien war ihnen untersagt. ${ }^{22}$

Bis 1842 ließen sich drei lizenzierte Geburtshelferinnen in der Stadt Biel nieder, zwei weitere folgten im Bezirk bis zur Jahrhundertmitte. ${ }^{23} \mathrm{Da}$ zumindest die Ersteren verheiratet oder verwitwet waren und zum Teil Kinder hatten ${ }^{24}$ dürfen wir den Hebammenberuf mit Eva Labouvie weniger als das Metier junger, ungebundener Frauen denn als das Geschäft gestandener Ehefrauen und Mütter begreifen, die auch bestrebt waren, mit ihrer Tätigkeit das familiäre Einkommen zu sichern. ${ }^{25}$ In Biel konnte sich hierfür aber keine von ihnen Hoffnung auf ein offizielles Amt samt Gehalt oder anderen Unterstützungsleistungen machen. Anders als etwa im Kanton Zürich waren bernische Gemeinden nicht gesetzlich verpflichtet, eine eigene Hebamme zur Versorgung der örtlichen Bevölkerung anzustellen. Zwar taten dies manche Kommunen und entschädigten ihre Geburtshelferinnen mit Sachleistungen oder «Wartgeldern», andere weigerten sich jedoch aktiv oder hielten die Einrichtung einer solchen Stelle für überflüssig. ${ }^{26} \mathrm{In}$ der Stadt Biel kam das Engagement für das Geburtswesen über wenige Reformversuche im 18. Jahrhundert offenbar nicht hinaus. Zwar regelte ein nach 1759 erlassener Hebammeneid ${ }^{27}$ formal die Aufgaben und Kompetenzen für Geburtshelferinnen, doch geschah dies wohl nicht mit Blick auf eine bestehende oder einzurichtende Stelle. Denn 1770 musste der Rat erkennen, «wie übel die stadt mit hebammen versehen» sei, und übertrug dem Ratsmitglied und späteren Stadt- oder Armenarzt Franz Ludwig Watt das neugeschaffene Amt eines «Accoucheurs». ${ }^{28}$ Dieser hatte es wohl bis zum Ende seiner Praxis 1801 inne. ${ }^{29}$ Welche Pflichten aber konkret mit dieser Aufgabe verbunden waren und ob auch die Stadtärzte des 19. Jahrhunderts eine

22 Siehe und vgl. Schneider/Emmert 1842, 43, 83, 84f.

23 Vgl. Schneider/Emmert 1842, 14, und StAB BB XI 326, 6.

24 Vgl. Schneider/Emmert 1842, 14. 1841 etwa behandelte Bloesch eine Tochter der Hebamme Rosina Streitmatter wegen Nasenblutens. Vgl. StadtA Biel, 4 A 1, Tagebuch, Bd. 20, 1841, 202. 25 Vgl. Labouvie 1999, 314.

26 Vgl. Meyer 1985, 51, und Schneider/Emmert 1842, 42, 79-83. Vgl. etwa für Zürich Bohner 1989, 10, 26-29.

27 Vgl. Bloesch/Weishaupt 2003, 651f.

28 Siehe ebd., 840.

29 Vgl. Bourquin/Bourquin 2008, Artikel «Watt», 476-477. 
ähnliche Doppelfunktion ausübten, geht aus den bislang ausgewerteten Quellen nicht hervor. ${ }^{30}$

Immerhin zeigen die Jahresrechnungen des städtischen Armengutes und jene der Spitalverwaltung, ${ }^{31}$ dass nur der Physikus fest im Dienst der Bieler Bürgerschaft stand, um die Gesundheitsfürsorge für deren bedürftige Mitglieder sicherzustellen. ${ }^{32}$ Die niedergelassenen Hebammen konnten zwar auch auf offiziell vermittelte Arbeitsgelegenheiten hoffen, etwa wenn nach Absprache mit dem Stadtarzt erkrankte Frauen im bielerischen Spital zu versorgen waren..$^{33}$ Ein regelmäßiges Einkommen ließ sich auf diesem Weg aber nicht erzielen, weshalb die private Praxis für sie einen umso höheren Stellenwert gehabt haben muss. Dies gilt auch für Cäsar Adolf Bloesch, dem es gelang, sich ohne die Übernahme einer offiziellen Stelle beruflich zu etablieren. ${ }^{34}$ Steigende Patienten- und Behandlungszahlen - insbesondere bei Frauen im gebärfähigen Alter - sprechen für eine zunehmende Akzeptanz bei der Klientel und dokumentieren die positive Entwicklung ihrer Nachfrage. Fand auch er im Feld der Geburtshilfe ein probates Mittel, sich unter den Mitbewerberinnen und Mitbewerbern am medizinischen Markt zu behaupten?

\section{Aspekte der «geburtshilflichen Praxis» Bloeschs}

Die möglichen Beweggründe, aus denen sich akademisch gebildete Mediziner in der ersten Hälfte des 19. Jahrhunderts der Geburtshilfe zuwandten,

30 Sowohl im Ratsbeschluss zur Einrichtung des Accoucheursamtes als auch in der Ordnung des Stadtphysikats sind vor allem Fragen der Vergütungen geregelt, Bestimmungen zur Berufsausübung fehlen hingegen. Vgl. Bloesch/Weishaupt 2003, 840f.

31 Vgl. StadtA Biel, BG Nr. 226 (für 1834, 1835, 1853), und ebd., 1,170, CLXXXIV, c. 1787-1821 (für 1787, 1790, 1820, 1821).

32 Stadtbewohner, die nicht den Bürgerstatus vorweisen konnten, waren von der Nutzung der bielerischen Gesundheitseinrichtungen ausgeschlossen. Erst ab 1850 wurden verarmte kranke Einwohner im Rahmen der freiwilligen Armenpflege auch zum Teil medizinisch unterstützt. Vgl. Helfer 1995, 29f. Zum Nebeneinander von Bürger- und Einwohnergemeinden im 19. Jahrhundert vgl. Kaestli 2011 für Biel und als Kurzüberblick für den Kanton Bern von Werdt 2011.

33 Außer zur Krankenpflege diente das Spital auch als Pfrund- und Armenhaus. Vgl. Bourquin/ Bourquin 2008, Artikel «Spitäler», 401-403. Die individuellen Erwerbschancen am Spital waren jedoch eher gering. 1820 etwa wurden hier 20 Personen längerfristig betreut. Die Hebamme Wisard kam in dieser Zeit einmal zum «visitieren» einer der Insassinnen, einer anderen verabreichte sie zwei Klistiere. Vgl. StadtA Biel, 1,170, CLXXXIV (1820), 1, 17.

34 Bloesch übernahm die Stadtarztstelle erst 1853, nachdem sein Vorgänger David Nieschang wegen nachlässiger Dienstführung in die Kritik geraten war. Auf das Jahresgehalt von 80 Franken - in etwa ein Fünftel des Jahreslohns eines Textilarbeiters - war Bloesch hingegen nicht mehr angewiesen: Er spendete es für wohltätige Zwecke. Vgl. StadtA Biel, BG Nr. 21 (1853), 15, 158; Gruner 1980, 141. 
waren weit gefächert. Das persönliche Interesse am Fach und seinen Herausforderungen konnte dafür sorgen, dass der einzelne Praktiker diesem Zweig der Medizin viel Aufmerksamkeit schenkte. Jakob Büchler etwa schrieb rückblickend auf sein langjähriges Engagement, dass er «die Geburtshülfe immer für einen sehr wichtigen Theil der Heilkunst» angesehen und sich ihr schon während des Studiums «mit einiger Vorliebe gewiedmet» habe. ${ }^{35}$ Unabhängig von ihren eigenen Einstellungen sahen sich zeitgenössische Mediziner aber auch mit den Ansprüchen der Klientel konfrontiert, die vom Arzt immer selbstverständlicher Fertigkeiten in der Obstetrik erwartete. ${ }^{36}$ Auch Bloesch dürfte sich dieses «Qualifikationsdrucks» bewusst gewesen sein, doch war er nach dem Bestehen des Examens vor dem kantonalen Sanitätskollegium im Juni 1827 für sein späteres Arbeiten gewappnet. Zwar beurteilte die Prüfungskommission seine Leistungen in Theorie und Praxis der Geburtshilfe sowie seine Demonstration am Phantom als «nicht so ausgezeichnet» wie diejenigen in den theoretischen Fächern. Das Gesamtergebnis, das nach Meinung der Vorsitzenden als «sehr günstig $»^{37}$ anzusehen war, wurde hiervon jedoch nicht beeinträchtig. Konnte Bloesch also mit seiner Qualifikation für innere Medizin, Chirurgie und Geburtshilfe mit den etablierten Bieler Kollegen gleichziehen, so entwickelte er im Lauf der Zeit einen eigenen Umgang mit dem dritten Berufsfeld. ${ }^{38}$

\section{Praktische Geburtshilfe bei Bloesch}

Da die Analysen über die in der Arztpraxis erreichten Behandlungszahlen oder die Geschlechterverhältnisse in der Patientenschaft noch andauern, können die vorläufigen Untersuchungsergebnisse zum Umfang der Geburtshilfe hier nicht mit diesen Parametern verglichen werden. Es sollte aber dennoch gelingen, sie grob in den Kontext der Gesamtpraxis zu stellen. Vom Juli 1832 bis Ende Dezember 1850 ließen sich insgesamt 52 Frauen aufgrund von Beschwerden behandeln, die im Kontext von Schwangerschaft, Geburt und Wochenbett auftraten. Vergleicht man diese Zahl mit den im Praxisjournal verzeichneten Patientinnen und Patienten, wird klar, dass Erstere nur eine kleine Minderheit in der ärztlichen Klientel bildeten. Zwischen 1832 und 1862 behandelte Bloesch insgesamt etwa 12000 Personen aus allen Altersgruppen. Die Zahl der im Journal bis 1850 verzeichneten Geburten fällt mit zwei sogar noch niedriger aus. Der erste Fall dieser Art datiert auf den 21. November 1840. Die Entbindung der Kaufmannsgattin Marie Elise Haag

35 Siehe Flückiger/Marthaler 2003, 134.

36 Vgl. Seidel 1998, $334 f$.

37 Siehe StAB, B XI 348, $18 f$.

38 Vgl. ebd. und Schneider/Emmert 1842, 4.

Gesnerus 69 (2012) 
dauerte bereits seit den Morgenstunden und gestaltete sich sehr schwierig. ${ }^{39}$ Die Bemühungen der Hebamme blieben bis zum Mittag erfolglos, und als die Lage «immer bedenklicher» wurde, rief man den jungen Arzt Karl Schaffter $^{40}$ für eine Zangengeburt. Sein Vorgehen verursachte bei der Mutter aber eher Schmerzen, als dass es die Geburt beförderte. Leider geht aus Bloeschs Aufzeichnungen nicht hervor, welche der anwesenden Personen ihn in dieser Situation schließlich hinzu rief. Offenbar verlangte man aber in erster Linie nur seinen Rat, denn Bloesch hielt sich auffallend im Hintergrund und konzentrierte sich vor allem auf die Ermahnung seines Kollegen, der Kreißenden mehr Zeit zu geben. Auch wenn er bei der zuletzt vorgenommenen inneren Wendung selbst einige Handgriffe ausführte ${ }^{41}$ - was den unglücklichen Ausgang nicht verhinderte - lag die führende Rolle bei dieser Geburt nicht bei ihm. ${ }^{42}$ Die zweite vermerkte Geburt fand 1844 statt. Obgleich es sich wieder um einen problematischen Fall handelte, fällt seine Beschreibung im Journal sehr viel kürzer und weniger detailliert aus. Constance Bichsel, die Ehefrau eines Notars, verspürte zwar seit mehr als einem Tag Wehen, doch ließ die Niederkunft beständig auf sich warten. ${ }^{43}$ Diese wurde laut Bloeschs Aufzeichnungen schließlich in der Nacht künstlich herbeigeführt. Nachdem die Fruchtblase instrumentell gesprengt worden war, habe man «die Zange appliciert u[nd] nach einer halben Stunde lebend ein[en] Knabe[n] zur Welt gebracht».44

Obwohl der Text einen erfolgreichen geburtshilflichen Eingriff dokumentiert und keine weiteren anwesenden Medizinalpersonen nennt, ist es zweifelhaft, ob Bloesch hier selbst tätig geworden ist. Denn selbst im Vergleich mit Ärzten, die die Obstetrik nicht zu ihrem Hauptgeschäft zählten, sind zwei dokumentierte Geburten in seiner weiblichen Klientel ein minimaler Wert. ${ }^{45}$ Vor allem die geringe praktische Erfahrung, die Cäsar Adolf Bloesch laut seinem Journal in diesen Dingen vorzuweisen hatte, macht seinen alleinigen Einsatz am Wochenbett einer Frau aus dem gehobenen städtischen Bürgertum äußerst unwahrscheinlich. Doch lässt sich die Dis-

39 Vgl. StadtA Biel, 4 A 1, Tagebuch, Bd. 18, 1840, 386-388.

40 Vgl. Mahlberg 2005, 54.

41 Vgl. StadtA Biel, 4 A 1, Tagebuch, Bd. 18, 1840, 386f.

42 Nach Bloeschs Ansicht wäre Nicht-Eingreifen in diesem Fall wohl die beste Option gewesen. Sich «selbst überlassen», urteilte er, hätten Mutter und Kind eventuell überlebt. Die Arbeit Dr. Schaffters sah er sehr kritisch und zeigte sich später überzeugt, dass «roher Kunst und einigen Fehlgriffen mit Recht die Schuld beigelegt werden dürfte». Vgl. StadtA Biel, 4 A 1, Tagebuch, Bd. 18, 1840, 413.

43 Vgl. StadtA Biel, 4 A 1, Tagebuch, Bd. 24, 1844, S. 444-446.

44 Vgl. ebd., 446.

45 Dies gilt beispielsweise für den Tiroler Landarzt Franz von Ottenthal. Vgl. den Beitrag von Marina Hilber in diesem Band. 
krepanz zwischen Journaleintrag und den quantitativen Ergebnissen mit einer alternativen Deutung auflösen. Da Bloesch den Zustand der Patientin vor der Entbindung zweimal kontrollierte und nur wenig Fortschritte im Geburtsverlauf feststellte, ${ }^{46}$ könnte er die künstliche Beendigung bereits einkalkuliert und die Familie von der Hinzuziehung eines Geburtshelfers überzeugt haben. In diesem Fall hätte er die Entbindung zwar vor Ort mitverfolgt und dabei vielleicht den allgemeinen Gesundheitszustand der Patientin überwacht, den Eingriff aber einem versierten Kollegen überlassen, wie es manche Hausärzte des 19. Jahrhunderts taten ${ }^{47}$ Eingehendere Untersuchungen über Bloeschs Verhältnis zu diesen beiden Patientinnen können eventuell klären, ob er für sie tatsächlich der primäre ärztliche Ansprechpartner im Sinne eines «Hausarztes» gewesen ist. Angesichts der beiden geschilderten Fälle lässt sich aber schon jetzt festhalten, dass Bloesch im untersuchten Zeitraum kein aktiver Geburtshelfer gewesen ist. Weder scheint sein Weg des Praxisaufbaus über diesen Bereich der Heilkunde geführt zu haben, noch hat er in späteren Jahren ein größeres Engagement entwickelt. Dieser Umstand war zu einem guten Teil sicherlich von der Einstellung des Arztes zur Obstetrik abhängig, auf die ich im folgenden Abschnitt näher eingehen möchte. Wenn wir jedoch Bloeschs geringe Aktivität nicht nur als Ergebnis seiner eigenen beruflichen Entscheidungen ansehen, sondern sie aus der Situation des medizinischen Marktes heraus zu erklären versuchen, erscheint ein weiteres Motiv als sehr wahrscheinlich. Demnach war Bloesch weder für die betroffenen Patientinnen noch für die anderen Heilkundigen eine qualifizierte Bezugsperson, die man bei tatsächlichen oder befürchteten Geburtskomplikationen gern zu Rate zog. Gerade Hebammen, denen im Notfall eine zentrale Rolle bei der Entscheidung für einen Geburtsarzt zukam, machten ihre Wahl stark von der Kompetenz der erreichbaren Ärzte abhängig. Mediziner, die im Entbindungsgeschäft wenig aktiv waren, hatten durch so eine Vermittlung natürlich das Nachsehen. ${ }^{48}$ Da entsprechende Quellen der übrigen Heilkundigen von Biel sowie solche aus der Patientenschaft fehlen, kann diese These leider nicht weiter untermauert werden. Sie macht aber nochmal deutlich, dass von Seiten des Arztes viel persönlicher Einsatz nötig war, um auch in anderen Bereichen als

46 Vgl. StadtA Biel, 4 A 1, Tagebuch, Bd. 24, 1844, 444f.

47 Vgl. Seidel 1998, 372. Eine solche überwachende Funktion bei einer nächtlichen Geburt würde auch den hohen Behandlungspreis von 16 Franken erklären. Vgl. StadtA Biel, 4 A 1, Tagebuch, Bd. 24, 1844, 446.

48 Vgl. Seidel 1998, 380-382. Im Praxisjournal konnten bislang nur sporadisch Hinweise auf Ärzte gefunden werden, die als Geburtshelfer aktiv waren. Neben dem schon genannten Karl Schaffter aus Biel wird auch Johann Knobel aus der Nachbarstadt Nidau genannt. Vgl. StadtA Biel, 4 A 1, Tagebuch, Bd. 18, 1841, 649, und Mahlberg 2005, 52. 
der inneren Medizin als Anbieter wahrgenommen und akzeptiert zu werden. Lassen sich auf Seiten Bloeschs Anzeichen hierfür finden? Und wenn es nicht seine Hilfe beim eigentlichen Geburtsgeschäft war, was erwarteten diejenigen Frauen von Bloesch, die ihn vor oder nach der Niederkunft aufsuchten? Erklärungsansätze für diese Fragen lassen sich in den Konsultationstexten der oben genannten 52 Patientinnen finden, auf die ich jetzt eingehen möchte.

\section{Geburtshilfe im Kontext der Allgemeinpraxis}

Schon als junger Arzt erfüllte C.A. Bloesch zwei Funktionen, die ins Profil eines geburtshilflich geschulten Arztes fielen: die des Ratgebers in gynäkologisch-obstetrischen Fragen und die als Therapeut von Krankheiten, welche mehr oder weniger direkt mit einer bestehenden oder beendeten Schwangerschaft verbunden waren. Gerade Frauen aus der städtischen Mittel- und Oberschicht scheinen sich über das korrekte Verhalten werdender Mütter und über Fragen zur Kinderpflege informiert zu haben. So erkundigte sich die Ehefrau des Bieler Gymnasiallehrers Binder 1833, ob sie als Schwangere «nach Stuttgart reisen dürfe, oder ob sie beßer thäte, zu warten bis nach ihrer Niederkunft». ${ }^{49}$ Bloesch bejahte, riet ihr aber zu einem gut gefederten Wagen und kurzen Reiseetappen. 1839 bat Maria Luise Funk, Ehefrau eines Advokaten, um Rat bei der Auswahl einer geeigneten Säugamme, da sie selbst nicht stillen wollte. Es sei wichtig, so die ärztliche Antwort, dass «in dem Alter der beiden Kinder kein großer Unterschied statt finde» ${ }^{50}$ und die Säugamme wie auch deren eigenes Kind gesund seien. Spiegelt sich in solchen Fragen neben der Sorge um das Wohl des Nachwuchses nicht zuletzt auch ein Interesse bürgerlicher Frauen, in der kindlichen Fürsorge den eigenen Status zu untermauern ${ }^{51}$ scheinen nichtbürgerliche Frauen Bloesch eher in echten Notlagen aufgesucht zu haben? Eine Bäuerin aus dem Dorf Murzelen gab zum Beispiel an, nach einem bedeutenden Schrecken keine Kindsbewegungen mehr spüren zu können, woraufhin ihr der Arzt Ruhe verordnete. ${ }^{52}$ Solch präventives Denken können wir zwar auch in jenen Krankheitsfällen vermuten, die die Patientinnen vor der Geburt ereilten und zu deren Behandlung Bloesch gerufen wurde.$^{53}$ Allerdings zeigen die Journaleinträge neben potentiell ernsthaften Beschwerden wie anhaltenden Blutflüssen vor der Geburt ${ }^{54}$ oft auch solche Leiden, die für Mutter und/oder

49 Siehe StadtA Biel, 4 A 1, Tagebuch, Bd. 4, 1833, 117.

50 Siehe StadtA Biel, 4 A 1, Tagebuch, Bd. 14, 1839, 5.

51 Vgl. hierzu Gestrich/Krause/Mitterauer 2003, 571-575.

52 Vgl. StadtA Biel, 4 A 1, Tagebuch, Bd. 35, 1850, 57.

53 Vgl. Seidel 1998, 401-403.

54 Vgl. zum Beispiel die Behandlung der Malersfrau Schüri: StadtA Biel, 4 A 1, Tagebuch, Bd. $30,1847,334$. 
Kind anscheinend nicht als bedenklich angesehen wurden. So rief ihn die eben genannte Friederike Binder vor der Entbindung nicht nur wegen der geplanten Reise, sondern auch je einmal wegen Übelkeit ${ }^{55}$ und wegen Zahnschmerzen. ${ }^{56}$ Nach der Niederkunft attestierte ihr der Arzt ein Milchfieber, außerdem hatte sie Schwierigkeiten beim Stillen. ${ }^{57}$

All diese medizinischen Leistungen konnten vom Arzt im Rahmen der alläglichen Hausbesuchs- und Sprechstundenpraxis erledigt werden und erforderten von ihm keinen großen zusätzlichen Aufwand. Anders sah es hingegen mit geburtshilflichen Fällen aus, die bedeutende Auswirkungen auf den ärztlichen Tagesablauf haben konnten. So hat Hans-Christoph Seidel mit Blick auf die Nachteile der obstetrischen Praxis herausgestellt, dass es gerade die «schwierige Integration der Geburtshilfe in die allgemeinärztliche Tätigkeit, ihr Zeitaufwand und der in Relation dazu beschränkte unmittelbare materielle Nutzen ${ }^{58}$ waren, die das Feld für viele Praktiker unattraktiv erscheinen ließen. Ein Fall aus dem Jahr 1842 mag verdeutlichen, dass auch Bloesch solche Bedenken trug und generell bemüht war, den regelmäßigen Gang seiner Praxis sicherzustellen - selbst dann, wenn sich ihm die Chance zur Betreuung einer Geburt bot. Am Morgen des 10. Oktober $1842 \mathrm{kam}$ die hochschwangere Magdalena Köhli in Bloeschs Praxis und klagte, dass sich am Vorabend Kreuzschmerzen und ein anhaltender Abgang von Blut und Schleim durch die Genitalien eingestellt hätten. ${ }^{59}$ Für ihren Arzt ließ das auf eine baldige Geburt schließen, so dass er seiner Patientin empfahl, «die Hebamme zu holen, sich ruhig zu halten u[nd] der Geburt abzuwarten». ${ }^{60}$ Wann dieser Zeitpunkt sein würde, darüber machte er keine Angaben. ${ }^{61}$ Indem er die Schwangere unter die Obhut der Hebamme stellte, zeigte der Arzt zwar, dass er die Situation durchaus ernst nahm. Er signalisierte aber auch, dass er nicht bereit war, nun selbst auf die Niederkunft zu warten. Am Nachmittag desselben Tages, nachdem er zwölf weitere Patienten besucht hatte, sah er nach der Köhli. Bloesch stellte keine grundsätzliche Änderung ihres Zustandes fest und verordnete ihr Ruhe, leichte Kost und ein Klistier. ${ }^{62}$ Auch in der folgenden Zeit machte Bloesch anscheinend keine

55 Vgl. StadtA Biel, 4 A 1, Tagebuch, Bd. 4, 1833, 74.

56 Vgl. StadtA Biel, 4 A 1, Tagebuch, Bd. 4, 1833, 200.

57 Vgl. StadtA Biel, 4 A 1, Tagebuch, Bd. 4, 1833, 249, 251.

58 Siehe Seidel 1998, 420. Vgl. auch Digby 1994, 269.

59 Dass es sich um einen Praxisbesuch handelte, schließe ich aus dem hierfür berechneten entsprechenden Tarif von einem halben Franken. Da alle folgenden Behandlungen im Hause Köhli stattfanden, sind hier die entsprechend höheren Hausbesuchstarife von 0.75 Franken notiert. Vgl. StadtA Biel, 4 A 1, Tagebuch, Bd. 22, 1842, 166.

60 Vgl. ebd.

61 Zur unsicheren Diagnostik von Schwangerschaft und zur ambivalenten Rolle von Prognosen vgl. Schlumbohm 2002.

62 Vgl. StadtA Biel, 4 A 1, Tagebuch, Bd. 22, 1842, 168. 
Anstalten, sich der Familie Köhli für die anstehende Entbindung zu empfehlen. Erst am übernächsten Tag erschien er wieder, notierte, dass die wehenartigen Schmerzen in den letzten 24 Stunden in Schüben wiedergekehrt sowie starke Kindsbewegungen zu fühlen seien. Die Blutungen hätten jedoch aufgehört. ${ }^{63}$ In der Nacht vom 13. auf den 14. Oktober wurde schließlich die Hebamme geholt und gegen Mittag ein gesundes Mädchen geboren. Bloesch begutachtete es zusammen mit der Mutter gegen Ende seines Arbeitstages bei der elften Behandlung an diesem Tag. ${ }^{64}$ Die Unwägbarkeiten der Geburtshilfe und insbesondere der hohe Zeitaufwand, so lässt sich an diesem Beispiel verdeutlichen, waren für Bloesch ebenso wie für viele andere Mediziner ein triftiger Grund, sich aus dem Entbindungsgeschäft herauszuhalten. Zu groß war die Gefahr, bei langer Abwesenheit andere Behandlungschancen zu verlieren oder die übrige Patientenschaft zu verstimmen. ${ }^{65}$ Zudem konnte kein Arzt sichergehen, für solche Wartezeiten angemessen entschädigt zu werden. Erst 1861 führte der Kanton eine Taxordnung für die Geburtshilfe ein, nach der Ärzten für eine künstliche Geburt 20 bis 80 Franken zustanden, ohne aber zu spezifizieren, mit welchem Zeitaufwand dies verbunden war. Dasselbe galt für Hebammen, denen zehn bis 25 Franken für «Hilfeleistung bei Geburt, sei diese leicht oder schwer, einfach oder mehrfach, inklusive Betreuung im Wochenbett bis zum 14. Tag» ${ }^{66}$ zustanden. Gegenüber den einmaligen und vergleichsweise hohen Einnahmen für Entbindungen zog auch Bloesch es vor, seiner Allgemeinpraxis die größte Aufmerksamkeit zu widmen. Auf lange Sicht nämlich hatte die Behandlung der Krankheiten von Schwangeren, Wöchnerinnen und Kindern eine hohe finanzielle Attraktivität. Allein die Behandlungskosten der oben genannten Magdalena Köhli vor und nach ihrer Entbindung beliefen sich bis zum Jahresende 1842 auf 11 Franken, was Bloeschs Honoraren für Hilfeleistungen bei Geburten schon recht nahe kam. ${ }^{67}$

63 Vgl. ebd., 171.

64 Vgl. ebd., 175.

65 Vgl. Seidel 1998, 333.

66 Zitiert nach Meyer 1985, 90.

$67 \mathrm{Zu}$ den Rechnungsbeträgen vgl. die Übersichten in StadtA Biel, 4 A 1, Tagebuch, Bd. 22, 205, 282, 367. Nach Durchsicht der Journaleinträge war Magdalena Köhli von Oktober bis Dezember das einzige Familienmitglied, das von Bloesch behandelt wurde. 


\section{Fazit}

Wie anhand des Beispiels Cäsar Adolf Bloeschs deutlich geworden ist, war die praktische Geburtshilfe ein Berufsfeld, dem sich akademische Ärzte des 19. Jahrhunderts oftmals nur unter großen Vorbehalten widmeten. Die geringe Anziehungskraft des Faches beruhte vor allem auf den befürchteten negativen Folgen, die das Engagement als Geburtshelfer für die Allgemeinpraxis als wichtigster Einkommensquelle haben konnte. Im Fall Bloeschs ließ sich anhand von Einträgen in seinem Praxisjournal zeigen, dass er sich mitunter aktiv vom Geburtsgeschäft fernhielt und schwangeren Patientinnen lieber eine Hebamme für die Niederkunft empfahl. Ein Zeichen, dass auch er entsprechende Vorbehalte gegenüber der Obstetrik hatte. Doch ist es möglich, dass neben seiner «Zurückhaltung» auch das Wahlverhalten der Patientinnen Bloeschs Stellung am Geburtsmarkt wesentlich beeinflusst hat. Wenn werdende Mütter oder Hebammen als deren reguläre erste Ansprechpersonen von der geburtshilflichen Kompetenz eines Arztes nicht überzeugt waren, unterblieb gewöhnlich auch deren Anstellung. Da aber weder das Praxisjournal noch andere Quellen Aufschluss über diese Entscheidungsprozesse geben, sind Aussagen über diesen Aspekt schwierig. Immerhin kann gesagt werden, dass auch der Bieler Arzt zumindest indirekt am lokalen Geburtsgeschehen partizipierte, indem er sich als Ratgeber und Therapeut von Leiden betätigte, die Schwangere und Wöchnerinnen ergriffen. Auf diesem Weg bot sich ihm auch eine Gelegenheit, die eigene Position am medizinischen Markt zu sichern.

\section{Archivsiglen}

StadtA Biel Stadtarchiv Biel

StAB Staatsarchiv Bern

\section{Quellen}

StadtA Biel, BG Nr. 21: Protokolle der Verhandlungen der Spital-Direktion der Stadt Biel vom 2. April 1849 bis 17. Dezember 1860

StadtA Biel, BG Nr. 226: Armengutsrechnungen 1798-1853

StadtA Biel, 1,170,CLXXXIV: Spitalrechnungen 1787-1821

StadtA Biel, 4 A 1, Tagebuch: Praxisjournal Cäsar Adolf Bloesch 1832-1863

StAB BB XI 326: Verzeichniß der Aerzte, Apotheker, und deren Gehülfen, und der Hebammen, im Kanton Bern, nach der Volkszählung von 1856

StAB B XI 348: Manual des Collegii Examinatii II. (1826-1834)

\section{Bibliographie}

Bloesch, Gustav/Achilles Weishaupt, Die Rechtsquellen der Stadt Biel mit ihren «̈̈usseren Zielen» Bözingen, Vingelz, und Leubringen (Basel 2003) 
Bohner, Brigitte Yvonne, Zur Ausbildung und Tätigkeit der Zürcher Hebammen im 19. Jahrhundert, Zürcher Medizingeschichtliche Abhandlungen 208 (Dietikon 1989)

Boucherin, Nadine, Die Krankengeschichten von C.A. Bloesch (1804-1863), in: Dietrich-Daum u.a. (Hrsg.), Arztpraxen im Vergleich: 18.-20. Jahrhundert (Innsbruck/Wien/Bozen 2008) 147-166

Bourquin, Werner/Marcus Bourquin, Biel. Stadtgeschichtliches Lexikon von der Römerzeit (Petinesca) bis Ende der 1930er Jahre (Biel 2008)

Büchler, Jakob, «Bericht über meine geburtshülfliche Praxis in den Jahren 1829-1839», in: Schweizerische Zeitschrift für Medicin, Chirurgie und Geburtshülfe 1 (1842) 97-106

Digby, Anne, Making a medical living. Doctors and patients in the English market for medicine, 1720-1910 (Cambridge 1994)

Ellerbrock, Dagmar, «Geschlecht, Gesundheit und Krankheit in historischer Perspektive», in: Hurrelmann, Klaus/Petra Kolip (Hrsg.), Geschlecht, Gesundheit und Krankheit in historischer Perspektive (Bern 2002) 118-141

Flückiger, Daniela/Stephanie Marthaler, Ärztliche Geburtshilfe im Berner Jura 1828-1861. Jakob Büchlers Berichte: Analyse-Diskussion-Edition (Diss. med. Bern 2003)

Gestrich, Andreas/Jens-Uwe Krause/Michael Mitterauer, Geschichte der Familie (Stuttgart 2003)

Green, Monica H., “Gendering the History of Women's Healthcare”, in: Gender \& History 20/3 (2008) 487-518

Gross, Dominik, «〈Deprofessionalisierung〉 oder 〈Paraprofessionalisierung〉? Die berufliche Entwicklung der Hebammen und ihr Stellenwert in der Geburtshilfe des 19. Jahrhunderts», in: Sudhoffs Archiv 82 (1998) 219-238

Gruner, Erich, Die Arbeiter in der Schweiz im 19. Jahrhundert: soziale Lage, Organisation, Verhältnis zu Arbeitgeber und Staat (Reprint München 1980)

Helfer, Monique, «Die Armut mit organisierter Wohltätigkeit bekämpfen. Armenwesen und öffentliche Fürsorge der Stadt Biel in der zweiten Hälfte des 19. Jahrhunderts», in: Bieler Jahrbuch (1995) 28-51

Hildebrand, Bruno (Hrsg.), Beiträge zur Statistik des Kantons Bern. I. Band. Die Bevölkerung. Erste Hälfte (Bern/Zürich 1860)

Huerkamp, Claudia, Der Aufstieg der Ärzte im 19. Jahrhundert. Vom gelehrten Stand zum professionellen Experten. Das Beispiel Preußens (Göttingen 1985)

Jenzer, Hans, «Die Gründung der Hebammenschulen in der Schweiz im 18. Jahrhundert (mit besonderer Berücksichtigung der bernischen Verhältnisse)», in: Gesnerus 23 (1966) 67-77

Kaestli, Tobias, «Die Bieler Stadtverwaltung seit 1815», in: Martig, Peter u.a. (Hrsg.), Berns moderne Zeit. Das 19. und 20. Jahrhundert neu entdeckt (Bern 2011) 79-82

Labouvie, Eva, Beistand in Kindsnöten: Hebammen und weibliche Kultur auf dem Land (1550-1910) (Frankfurt a.M. 1999)

Mahlberg, Daniel Alexander, Der ärztliche Bezirksverein Bern-Seeland: 1845-1895 (Diss. med. dent. Bern 2005)

Meyer, Katharina, Zur Geschichte des Hebammenwesens im Kanton Bern, Berner Beiträge zur Geschichte der Medizin und der Naturwissenschaften 11 (Bern 1985)

Niederhäusern, Raffael von, «Die Gesellschaftsstruktur der Stadt Biel Mitte des 19. Jahrhunderts», in: Bieler Jahrbuch (2011) 71-88

Pfister, Christian/Hans-Rudolf Egli (Hrsg.), Historisch-statistischer Atlas des Kantons Bern, 1750-1995: Umwelt, Bevölkerung, Wirtschaft, Politik (Bern 1998)

Porter, Dorothy/Roy Porter, Patient's Progress. Doctors and Doctoring in Eighteenth-century England (Stanford 1989)

Rieder, Philipp/Micheline Louis-Courvoisier, "Enlightened Physicians: Setting Out on an Elite Academic Career in the Second Half of the Eighteenth Century", in: Bulletin of the History of Medicine 84 (2010) 578-606

Schlumbohm, Jürgen, «Grenzen des Wissens: Verhandlungen zwischen Arzt und Schwangeren im Entbindungshospital der Universität Göttingen um 1800», in: Duden, Barbara/Jürgen Schlumbohm/Patrice Veit (Hrsg.), Geschichte des Ungeborenen. Zur Erfahrungs- und Wissenschaftsgeschichte der Schwangerschaft, 17.-20. Jahrhundert (Göttingen 2002) 129-166

Schneider, Johann Rudolf/Friedrich Carl Emmert, Systematische Sammlung der berner'schen Medicinalgesetze, Dekrete und Verordnungen (Bern 1842)

124 Gesnerus $69(2012)$ 
Schulz, Stefan, «Wege in die Klinik. Anmerkungen zur Geschichte der Geburtshilfe», in: Ley, Astrid/Marion Maria Ruisinger (Hrsg.), Von Gebärhaus und Retortenbaby. 175 Jahre Frauenklinik Erlangen. Begleitband zur Ausstellung im Stadtmuseum Erlangen (Nürnberg 2003) 14-31

Seidel, Hans-Christoph, Eine neue 〈Kultur des Gebärens〉. Die Medikalisierung von Geburt im 18. und 19. Jahrhundert in Deutschland (Stuttgart 1998)

Werdt, Christophe von, «Der Dualismus von Burger- und Einwohnergemeinden», in: Martig, Peter u.a.(Hrsg.), Berns moderne Zeit. Das 19. und 20. Jahrhundert neu entdeckt (Bern 2011) 93-96 